\title{
Pullback Attractors for Nonautonomous Degenerate Kirchhoff Equations with Strong Damping
}

\author{
Honglv Ma $\mathbb{D},{ }^{1}$ Jing Wang $\mathbb{D}^{1},{ }^{1}$ and Jun Xie $\mathbb{C}^{2}$ \\ ${ }^{1}$ School of Mathematics, Southeast University, Nanjing 211189, China \\ ${ }^{2}$ College of Electronic Engineering, Nanjing Xiaozhuang University, Nanjing 211171, China
}

Correspondence should be addressed to Honglv Ma; honglvma@seu.edu.cn

Received 31 July 2021; Accepted 15 November 2021; Published 20 December 2021

Academic Editor: Sergey Shmarev

Copyright (C) 2021 Honglv Ma et al. This is an open access article distributed under the Creative Commons Attribution License, which permits unrestricted use, distribution, and reproduction in any medium, provided the original work is properly cited.

In this paper, we obtain the existence of pullback attractors for nonautonomous Kirchhoff equations with strong damping, which covers the case of possible generation of the stiffness coefficient. For this purpose, a necessary method via "the measure of noncompactness" is established.

\section{Introduction}

Let $\Omega \subset \mathbb{R}^{n}$ be a bounded domain with smooth boundary $\partial \Omega$. We consider the following Kirchhoff wave model with strong damping:

$$
\left(\begin{array}{ll}
u_{t t}-\Delta u_{t}-\phi\left(\|\nabla u\|^{2}\right) \Delta u+f(u)=h(x, t), & \text { in } \Omega \times(\tau, \infty), \\
\left.u\right|_{\partial \Omega}=0, u(x, \tau)=u_{\tau}^{0}(x), u_{t}(x, \tau)=u_{\tau}^{1}(x), & x \in \Omega, \tau \in \mathbb{R},
\end{array}\right.
$$

where $h(x, t)$ is a time-dependent external force term, $u_{\tau}^{0}$ and $u_{\tau}^{1}$ are initial data, and $\phi$ and $f$ are nonlinear functions specified later.

To describe small vibrations of an elastic stretched string, Kirchhoff [1] introduced the equation

$$
\rho h \frac{\partial^{2} u}{\partial t^{2}}=\left\{p_{0}+\frac{E h}{2 L} \int_{0}^{L}\left(\frac{\partial u}{\partial x}\right)^{2} d x\right\} \frac{\partial^{2} u}{\partial x^{2}}+g
$$

where $u=u(x, t)$ is the lateral deflection, $0<x<L$ the space coordinate, $t \geq 0$ the time, $E$ the Young's modulus, $\rho$ the mass density, $h$ the cross-section area, $L$ the length, $p_{0}$ the initial axial tension, and $g$ the external force. It has been called the Kirchhoff equation since then. In general, we call the Kirchhoff equation nondegenerate if the stiffness $\phi$ sat- isfies the strict hyperbolicity condition $\phi(s) \geq c>0$ and degenerate if $\phi(s) \geq 0$ on $\mathbb{R}^{+}$. Obviously, the degenerate stiffness coefficient $\phi(s)$ in (1) corresponds to the case that the initial axial tension equals zero.

From the mathematical point of view, global existence of the model like (2) has been proven in a multitude of special situations in $\Omega \subset \mathbb{R}^{n}$. We refer to [2-5] for the analytic data, [6-9] for the dispersive equations and small data, and [10-15] for the weak damped equations.

Introducing the strong damping term $-\Delta u_{t}$ provides an additional a priori estimate. Certainly, from the physical point of view, the dissipative plays an important spreading role for the energy gathered arising from the nonlinearity in a real process. Concerning Kirchhoff equations with strong dissipation, the first result on the well-posedness we are aware of was obtained by Nishihara [16]. He proved the global existence of the solution for the model $u_{t t}-\Delta u_{t}$ $-m(\|\nabla u\|) \Delta u=0$. In recent years, many mathematicians and physicists paid their attentions to this type of problem and obtained the well-posedness under different types of hypotheses, such as the absent source term [17] and the subcritical source term [18-23]. In general, the exponent $p^{*}=$ $n+2 /(n-2)^{+}$is called to be critical when someone studies the problem in $\left.H_{0}^{1}(\Omega)\right) \times L^{2}(\Omega)$. Assuming the stiffness factor is nondegenerate $\left(\phi(s) \geq \phi_{0}>0\right)$, References [18-24] also proved the existence of the attractor. In the case of possible degeneration of the stiffness coefficient and the case of 
supercritical source term $\left(p^{*}<p<(n+4) /(n-4)^{+}\right)$, the first result on the well-posedness we are aware of is given by Chueshov [25]. However, when he proved the existence of a global attractor for problem (1) in the natural energy space $\left(H_{0}^{1}(\Omega) \cap L^{p+1}(\Omega)\right) \times L^{2}(\Omega)$ endowed with a partially strong topology (in the sense, if $\left(u_{0}^{n}, u_{1}^{n}\right) \longrightarrow\left(u_{0}, u_{1}\right)$ with a partially strong topology, then $\left(u_{0}^{n}, u_{1}^{n}\right) \longrightarrow\left(u_{0}, u_{1}\right)$ strongly in $H_{0}^{1} \times L^{2}$ and $u_{0}^{n} \rightarrow u_{0}$ weakly in $\left.L^{\mathrm{p}+1}\right)$, he assumed that

$$
\phi(s)>0, \quad \forall s \geq 0, \phi \in C^{1}\left(\mathbb{R}^{+}\right) .
$$

Under this condition, one can conclude that $\phi($ $\left.\|\nabla u(t)\|^{2}\right) \geq c_{0}>0$ if $\|\nabla u(t)\|$ is bounded for $t \in \mathbb{R}^{+}$. Recently, $\mathrm{Ma}$ et al. [26] proved the existence of the global attractor in the case of degeneration for the autonomous Kirchhoff system.

The pullback attractor is a basic concept to study the longtime dynamics of nonautonomous evolution equations (see [27-32] and references therein). It is worth mentioning that there are only a few recent results devoted to the pullback attractor for nonautonomous systems like (1). In 2013, Wang and Zhong [33] investigated the upper semicontinuity of pullback attractors for problem (1) with $\phi(s)=1$ $+\varepsilon s(\varepsilon>0)$ and $\left|f^{\prime}(u)\right| \leq C\left(|u|^{2 /(n-4)^{+}}+1\right)(n \geq 3)$. Recently, $\mathrm{Li}$ and Yang [34] studied the robustness of pullback attractors with $\phi^{\prime}(s) \geq 0, \phi(0)=\phi_{0}>0$. We notice that all these publications assume that the stiffness factor is nondegenerate, or more precisely, $\phi(0)>0$ and $\phi$ is nondecreasing.

In this paper, we consider the problem (1) under the degenerate hyperbolicity condition $\phi(s) \geq 0$. We do not assume that $\phi$ is monotone and allow $\phi(0)=0$, such as $\phi(s$ )$=b s^{\gamma}$ (degenerate and monotone) or $\phi(s)=\left(1+\sin ^{2} s\right) s^{\gamma}$ (degenerate and nonmonotone) with $\gamma \geq 1$. Based on the result in $[25,26]$, we prove the existence of pullback attractors in $\left.H_{0}^{1}(\Omega)\right) \times L^{2}(\Omega)$ if $\phi$ is really degenerate. To overcome the difficulties caused by the degeneration, we first established a method (condition (D-PC)) via "the measure of noncompactness" (some ideas come from $[35,36]$ ) to prove that the process is pullback $\mathscr{D}$-asymptotically compact.

The paper is organized as follows. In Section 2, we introduce some preliminaries and establish a necessary abstract result (see Theorem 5). In Section 3, we discuss the existence of pullback attractors for the equation (1) (see Theorem 12).

\section{Preliminaries}

In this section, we will give some notations and results. As usual, we denote by $\|\cdot\|$ and $(\cdot, \cdot)$ the norm and the inner product in $L^{2}(\Omega)$, respectively. Let $\mathscr{H}=H_{0}^{1}(\Omega) \times L^{2}(\Omega)$. We define the norms in $\mathscr{H}$ by $\left\|u_{0}, u_{1}\right\|_{\mathscr{H}}^{2}=\left\|\nabla u_{0}\right\|^{2}+\left\|u_{1}\right\|^{2}$.

Let $X$ be a Banach space and $U(t, \tau)$ be a process acting on $X$. In the following, we recall some definitions and results related to the pullback attractors; more details can be found in $[27,29,33]$.
Definition 1. A family of compact sets $\mathscr{A}=\{A(t)\}_{t \in \mathbb{R}}$ is said to be a pullback attractor for process $U(\cdot, \cdot)$ if

(i) $\mathscr{A}$ is invariant, that is, $U(t, \tau) A(\tau)=A(t)$, for all $t \geq \tau$

(ii) $\mathscr{A}$ is pullback attracting, i.e., $d(U(t, t-\tau) B, A(t))$ $\longrightarrow 0$, as $\tau \longrightarrow+\infty$, for all bounded subset $B$ of $X$, where $d(B, A)$ is the Hausdorff semidistance

Definition 2. A family of sets $\mathscr{D}=\{D(t)\}_{t \in \mathbb{R}}$ is said to be a pullback absorbing family for process $U(\cdot, \cdot)$, if for all $t \in$ $\mathbb{R}$ and all bounded $B \subset X$, there exists $T=T(t, B)>0$, such that $U(t, t-\tau) B \subset D(t)$, for all $\tau \geq T$. In addition, the family $\mathscr{D}$ is said to be pullback $\mathscr{D}$-absorbing, if for any $t \in \mathbb{R}$, there exists $T_{t}>0$ such that $U(t, t-\tau) D(t-\tau) \subset D(t)$ for $\tau \geq T_{t}$.

Definition 3. A process $U(\cdot, \cdot)$ is said to be pullback $\mathscr{D}$ -asymptotically compact in $X$, if for any $t \in \mathbb{R}$, any sequences $\tau_{n} \longrightarrow \infty$ and $x_{n} \in D\left(t-\tau_{n}\right) ; \quad$ the sequence $\left\{U\left(t, t-\tau_{n}\right) x_{n}\right\}_{n \in \mathbb{N}}$ is relatively compact in $X$.

Lemma 4 (see [29]). Let the family $\mathscr{D}=\{D(t)\}_{t \in \mathbb{R}}$ be pullback absorbing and $U(\cdot, \cdot)$ be continuous and pullback D-asymptotically compact in X. Then, the family $\mathscr{A}=$ $\{A(t)\}_{t \in \mathbb{R}}$ defined by

$$
A(t)=\bigcap_{s \geq 0} \bigcup_{\tau \geq s} U(\bar{t}, t \tau) D(t \tau)
$$

is a pullback attractor for $U(\cdot, \cdot)$.

To verify the pullback $\mathscr{D}$-asymptotically compact property in $X$, it suffices to check the following condition.

2.1. D-Pullback Condition (D-PC). For any $\delta>0$ and $t \in \mathbb{R}$, there exist $\tau_{0}=\tau_{0}(t, \mathscr{D}, \delta)>0$ and a finite dimensional space $X_{1}$ of $X$ such that

$$
\begin{aligned}
& \left\{P\left(U\left(t, t-\tau_{0}\right) D\left(t-\tau_{0}\right)\right)\right\} \text { is bounded, } \\
& \quad\left\|(I-P)\left(U\left(t, t-\tau_{0}\right) x\right)\right\|_{X}<\delta, \quad \forall x \in D\left(t-\tau_{0}\right),
\end{aligned}
$$

where $P: X \longrightarrow X_{1}$ is a bounded projector.

Theorem 5. Let the family $\mathscr{D}=\{D(t)\}_{t \in \mathbb{R}}$ be a pullback $\mathscr{D}$ -absorbing family of the process $U(t, \tau)$. If the D-pullback condition (D -PC) holds, then $U(\cdot, \cdot)$ is pullback $\mathscr{D}$-asymptotically compact in $X$.

Proof. By Definition 3, the result will be proven if we can show that for any $t \in \mathbb{R}$, any sequences $\tau_{n} \longrightarrow \infty$ and $x_{n} \in$ $D\left(t-\tau_{n}\right),\left\{U\left(t, t-\tau_{n}\right) x_{n}\right\}_{n \in \mathbb{N}}$ is relatively compact in $X$.

For every $\delta>0$, condition (D-PC) implies that there exist $\tau_{0}=\tau_{0}(t, \mathscr{D}, \delta)>0$ and the finite dimensional space $X_{1}$, such that (5) holds. Then, we have

$$
\begin{aligned}
& \gamma\left(U\left(t, t-\tau_{0}\right) D\left(t-\tau_{0}\right)\right) \leq \gamma\left[P\left(U\left(t, t-\tau_{0}\right) D\left(t-\tau_{0}\right)\right)\right] \\
& \quad+\gamma\left[\left((I-P)\left(U\left(t, t-\tau_{0}\right) D\left(t-\tau_{0}\right)\right)\right)\right] \leq \gamma(N(0, \delta)) \leq 2 \delta
\end{aligned}
$$


where $\gamma$ is the measure of noncompactness defined as $\gamma(B)=\inf \{\delta>0 \mid B$ admits a finite cover by sets whose diameter $\leq \delta\}$.

On the other hand, the properties of $\mathscr{D}$ give that there exists $T_{t-\tau_{0}}>0$, such that for $\tau \geq T_{t-\tau_{0}}, U\left(t-\tau_{0}, t-\tau_{0}-\tau\right)$ $D\left(t-\tau_{0}-\tau\right) \subset D\left(t-\tau_{0}\right)$ and

$$
\begin{aligned}
& U\left(t, t-\tau_{0}-\tau\right) D\left(t-\tau_{0}-\tau\right) \\
& =U\left(t, t-\tau_{0}\right) U\left(t-\tau_{0}, t-\tau_{0}-\tau\right) D\left(t-\tau_{0}-\tau\right) \\
& \quad \cdot \subset U\left(t, t-\tau_{0}\right) D\left(t-\tau_{0}\right)
\end{aligned}
$$

Then, we can find $N_{0}$, such that $\left.\gamma\left(\bigcup_{n>N_{0}} U\left(t, t-\tau_{n}\right) x_{n}\right)\right)$ $\leq 2 \delta$, which means that $\left\{U\left(t, t-\tau_{n}\right) x_{n}\right\}_{n \in \mathbb{N}}$ has a finite $4 \delta$ -net for any $\delta>0$. The proof is complete.

\section{Existence of Pullback Attractors}

In this section, we will prove the existence of the pullback attractor when $\phi(s)$ is really degenerate and $f(u)$ is subcritical. We assume that $f, \phi$, and $h$ satisfy the following conditions.

Assumption 6. The function $\phi \in C^{1}\left(\mathbb{R}^{+}\right), \phi(s) \geq \min \left\{L_{1} s^{\alpha}\right.$, $\left.L_{2}\right\}$ for $s \in \mathbb{R}^{+}$, and some constants $\alpha \geq 0, L_{1}, L_{2}>0$. Moreover, there exists $\delta_{0}>0$ such that

$$
\liminf _{s \longrightarrow+\infty}\left(s \phi(s)-\delta_{0} \Phi(s)\right)>-\infty
$$

where $\Phi(s)=\int_{0}^{s} \phi(t) \mathrm{d} t$.

Assumption 7. $f(u)$ is a $C^{1}$ function, $f(0)=0, f^{\prime}(s) \geq-c_{1}$, and $s \in \mathbb{R}$,

$$
\mu_{f}=\liminf _{|s| \longrightarrow+\infty} \frac{f(s)}{s}>-\lambda_{1} \phi_{\infty} \quad \text { with } \phi_{\infty}=\liminf _{s \longrightarrow+\infty} \phi(s),
$$

and the following properties hold:

(i) if $n=1$, then $f$ is arbitrary

(ii) if $n=2$, then

$$
\left|f^{\prime}(u)\right| \leq C\left(1+|u|^{p-1}\right) \text { with } 1 \leq p<\infty \text {, }
$$

(iii) if $n \geq 3$, then

$$
\left|f^{\prime}(u)\right| \leq C\left(1+|u|^{p-1}\right) \text { with } 1 \leq p<p_{*}=\frac{n+2}{n-2}
$$

where $c_{1}$ and $C$ are positive constants and $\lambda_{1}$ is the first eigenvalue of $-\Delta$.

Assumption 8. $h, \partial_{t} h \in L_{\text {loc }}^{2}\left(\mathbb{R}, L^{2}(\Omega)\right)$ and

$$
\int_{-\infty}^{t}\|h(\cdot, s)\|^{2} \mathrm{~d} s<+\infty, \quad \forall t \in \mathbb{R}
$$

Remark 9. (1) $\phi(s)=L_{1} s^{\alpha}$ or $\widetilde{\phi}(s)=\left(1+\sin ^{2} s\right) s^{\alpha}(\alpha \geq 1)$ satisfies Assumption 6. It indicates that we include into the consideration the case of possibly degenerate $\phi$ since $\phi(0)=0$. Moreover, because $\phi_{\infty}=+\infty$ in this case, $\mu_{f}>-$ $\lambda_{1} \phi_{\infty}$ becomes $\mu_{f}>-\infty$. If $\alpha=0$, then $\phi(s)$ is a constant, and equation (1) is the nonlinear wave equation with strong damping.

(2) Assumptions 6 and 7 imply that there exist constants $c_{0}>0, \theta_{1}>0$ with $0<\phi_{1}<\phi_{\infty}, 0<\phi_{1} \lambda_{1}-\theta_{1}<<1$ such that

$$
\Phi(s) \geq \phi_{1} \cdot s-c_{0} \phi_{1} \quad \forall s \in \mathbb{R}^{+}
$$

$$
\begin{aligned}
F(s) & \geq-\frac{\theta_{1}}{2} s^{2}-c_{2}, f(s) s \geq-\theta_{1} s^{2}-c_{2}, f(s) s-F(s) \\
& \geq-\frac{c_{1}}{2} s^{2}, \quad \forall s \in \mathbb{R}
\end{aligned}
$$

where $F(s)=\int_{0}^{s} f(t) \mathrm{d} t$.

The well-posedness of the problem

$$
\left(\begin{array}{l}
\partial_{t t} u-\sigma\left(\|\nabla u\|^{2}\right) \Delta \partial_{t} u-\phi\left(\|\nabla u\|^{2}\right) \Delta u+f(u)=h(x), \quad \text { in } \Omega \times(0, \infty), \\
\left.u\right|_{\partial \Omega}=0, u(x, 0)=u^{0}(x), u_{t}(x, 0)=u^{1}(x), \quad x \in \Omega,
\end{array}\right.
$$

has been established by Chueshov [25] in the autonomous case. Noticing that the conditions of $\phi, f$ are more general than the above Assumptions 6-8, we can obtain the following Proposition 10 by a similar argument as in [25], except for the treatment of $h(x, t)$. The reader is referred to the Appendix for a detailed proof of these facts.

Proposition 10. Let Assumptions 6-8 be in force. Then, for $\tau, T \in \mathbb{R}(\tau<T)$ and $\left(u_{\tau}^{0}, u_{\tau}^{1}\right) \in \mathscr{H}$, problem (1) has a unique weak solution $u$ with $\left(u, u_{t}\right) \in C([\tau, T] ; \mathscr{H})$ and

(1) for every $t \in[\tau, T]$, there exists $C=C_{R, \tau, T}>0$ such that

$$
\left\|u_{t}(t)\right\|^{2}+\|\nabla u(t)\|^{2}+\int_{\tau}^{t}\left\|\nabla u_{t}(s)\right\|^{2} d s \leq C,
$$

$$
\begin{aligned}
& E\left(u(t), u_{t}(t)\right)+2 \int_{s}^{t}\left(\left\|\nabla u_{t}(r)\right\|^{2}-\left(h, u_{t}\right)\right) d r \\
& =E\left(u(s), u_{t}(s)\right), \tau \leq s<t
\end{aligned}
$$

where $E\left(u_{0}, u_{1}\right)=\left\|u_{1}\right\|^{2}+\Phi\left(\left\|\nabla u_{0}\right\|^{2}\right)+2 \int_{\Omega} F\left(u_{0}\right) d x$, $\left\|\left(u_{\tau}^{0}, u_{\tau}^{1}\right)\right\|_{\mathscr{H}} \leq R$

(2) for every $t \in(\tau, T]$, there exists $K=K_{R, \tau, T}>0$ such that

$$
\begin{aligned}
& \left\|u_{t t}(t)\right\|_{H^{-1}}^{2}+\left\|\nabla u_{t}(t)\right\|^{2} \\
& \leq K\left(1+\frac{1}{(t-\tau)^{2}}\right)\left(1+\int_{\tau}^{T}\left(\|h(\cdot, s)\|^{2}+\left\|h_{t}(\cdot, s)\right\|^{2}\right) d s\right) .
\end{aligned}
$$


(3) the Lipschitz stability

$$
\|\left(z(t), z_{t}(t)\left\|_{\mathscr{H}}^{2} \leq K\right\|\left(z(\tau), z_{t}(\tau) \|_{\mathscr{H}}^{2},\right.\right.
$$

holds for $z(t)=u^{1}(t)-u^{2}(t)$, where $u^{1}, u^{2}$ are two weak solutions of problem (1) with initial data $\left(u_{i, \tau}^{0}, u_{i, \tau}^{1}\right)$, $\left\|\left(u_{i, \tau}^{0}, u_{i, \tau}^{1}\right)\right\|_{\mathscr{H}} \leq R, i=1,2$.

We define the solution operator $U(t, \tau): \mathscr{H} \longrightarrow \mathscr{H}$ associated to problem (1) as

$$
U(t, \tau)\left(u_{\tau}^{0}, u_{\tau}^{1}\right)=\left(u(t), u_{t}(t)\right), \quad \forall t \geq \tau, \tau \in \mathbb{R},
$$

where $u$ is the weak solution of problem (1) corresponding to initial data $\left(u_{\tau}^{0}, u_{\tau}^{1}\right) \in \mathscr{H}$. Then, we know from Proposition 10 that $U(t, \tau): \mathscr{H} \longrightarrow \mathscr{H}$ is a continuous evolution process. For convenience, we denote by $\xi_{u}(t)=\left(u(t), u_{t}(t)\right)$ for any function $u(t)$. As $\left(u(\tau), u_{t}(\tau)\right)=\left(u_{\tau}^{0}, u_{\tau}^{1}\right)$, we also denote $\left(u_{\tau}^{0}, u_{\tau}^{1}\right)$ by $\xi_{u}(\tau)$.

Lemma 11. Let Assumptions 6-8 be valid. Then, the process $U(\cdot, \cdot)$ defined in (21) has a pullback D-absorbing family $\mathscr{D}=\{D(t)\}_{t \in \mathbb{R}}$. Moreover, $D(t)$ is bounded in $\mathscr{H}_{1}=H_{0}^{1}(\Omega)$ $\times H_{0}^{1}(\Omega)$ for every $t \in \mathbb{R}$.

Proof. As usual, the argument below can be justified by considering Galerkin approximations. Using the multiplier $u_{t}$ $+\eta u$ in Equation (1), we have that

$$
\frac{\mathrm{d}}{\mathrm{d} t} W^{\eta}\left(\xi_{u}(t)\right)+K\left(\xi_{u}(t)\right)=0, \quad t \geq \tau,
$$

where

$$
\begin{aligned}
W^{\eta}\left(\xi_{u}(t)\right)= & \left\|u_{t}\right\|^{2}+\Phi\left(\|\nabla u\|^{2}\right)+2(F(u), 1) \\
& +\eta\left[\|\nabla u\|^{2}+2\left(u_{t}, u\right)\right] \geq(1-\eta)\left\|u_{t}\right\|^{2} \\
& +\phi_{1} \cdot\|\nabla u\|^{2}-c_{0} \phi_{1}-\theta_{1}\|u\|^{2}-2 c_{2} \cdot \text { mes } \Omega \\
& +\eta\|\nabla u\|^{2}-\eta\|u\|^{2} \geq \kappa\left\|\xi_{u}(t)\right\|_{\mathscr{H}}^{2}-C_{3},
\end{aligned}
$$

$$
\begin{aligned}
K\left(\xi_{u}(t)\right)= & 2\left\|\nabla u_{t}\right\|^{2}-2 \eta\left\|u_{t}\right\|^{2}+2 \eta\left[\phi\left(\|\nabla u\|^{2}\right)\|\nabla u\|^{2}\right. \\
& +(f(u), u)]-2\left(h, u_{t}+\eta u\right),
\end{aligned}
$$

for $\eta>0$ which is small enough, $\kappa>0$ is a positive constant, and $\kappa, C_{3}$ are independent of $\xi_{u}(t)$. that

Since Assumption 6 implies that there exists $L_{3}>0$ such

$$
\delta_{0} \Phi\left(\|\nabla u\|^{2}\right) \leq \phi\left(\|\nabla u\|^{2}\right)\|\nabla u\|^{2}+L_{3}
$$

combining with (15), we have that

$$
\begin{aligned}
& W^{\eta}\left(\xi_{u}(t)\right) \leq(1+\eta)\left\|u_{t}\right\|^{2}+\Phi\left(\|\nabla u\|^{2}\right)+2(f(u), u) \\
& \quad+c_{1}\|u\|^{2}+\eta\left[\|\nabla u\|^{2}+\|u\|^{2} \leq \kappa_{1}\left\|\xi_{u}(t)\right\|_{\mathscr{H}}^{2}+\Phi\left(\|\nabla u\|^{2}\right)\right. \\
& \quad+2(f(u), u) \leq \kappa_{1}\left\|\xi_{u}(t)\right\|_{\mathscr{H}}^{2}+\Phi\left(\|\nabla u\|^{2}\right)+C_{3}\|u\|_{L^{p+1}}^{p+1}+C_{4},
\end{aligned}
$$

$$
\begin{aligned}
& K\left(\xi_{u}(t)\right) \geq\left\|\nabla u_{t}\right\|^{2}+\left(\lambda_{1}-2 \eta\right)\left\|u_{t}\right\|^{2}+(2 \eta-\varepsilon) \phi\left(\|\nabla u\|^{2}\right)\|\nabla u\|^{2} \\
& \quad+\varepsilon\left(\delta_{0} \Phi\left(\|\nabla u\|^{2}\right)-L_{3}\right)+2 \eta(f(u), u)-\delta\left\|u_{t}\right\|^{2}-\delta \eta^{2}\|u\|^{2} \\
& \quad-\frac{2}{\delta}\|h(\cdot, t)\|^{2} .
\end{aligned}
$$

Then, we can find $\eta>0, \varepsilon>0, \delta>0$ small enough such that

$$
\begin{aligned}
& K\left(\xi_{u}(t)\right)-\delta W^{\eta}\left(\xi_{u}(t)\right) \geq\left\|\nabla u_{t}\right\|^{2}+\left(\lambda_{1}-2 \eta-\delta(1+\eta)-\delta\right)\left\|u_{t}\right\|^{2} \\
& \quad+(2 \eta-\varepsilon) \phi\left(\|\nabla u\|^{2}\right)\|\nabla u\|^{2}+\varepsilon \delta_{0} \Phi\left(\|\nabla u\|^{2}\right) \\
& \quad-\varepsilon L_{3}+2(\eta-\delta)(f(u), u)-c_{1} \delta\|u\|^{2}-\delta \Phi\left(\|\nabla u\|^{2}\right) \\
& \quad-\delta \eta\|\nabla u\|^{2}-\delta\left(\eta^{2}+\eta\right)\|u\|^{2}-\frac{2}{\delta}\|h(\cdot, t)\|^{2} \geq\left\|\nabla u_{t}\right\|^{2} \\
& \quad+(2 \eta-\varepsilon)\left(\phi_{1} \cdot\|\nabla u\|^{2}-c_{0} \phi_{1}\right)+\left(\varepsilon \delta_{0}-\delta\right) \Phi\left(\|\nabla u\|^{2}\right) \\
& \quad-2 \eta \frac{\theta_{1}}{\lambda_{1}}\|\nabla u\|^{2}-\delta \eta\|\nabla u\|^{2}-\delta\left(c_{1}+\eta^{2}+\eta\right)\|u\|^{2}-\frac{2}{\delta}\|h(\cdot, t)\|^{2} \\
& -C_{4} \geq\left\|\nabla u_{t}\right\|^{2}-C\left(1+\|h(\cdot, t)\|^{2}\right) .
\end{aligned}
$$

By (22) and (28), we get that

$$
\frac{\mathrm{d}}{\mathrm{d} t} W^{\eta}\left(\xi_{u}(t)\right)+\delta W^{\eta}\left(\xi_{u}(t)\right)+\left\|\nabla u_{t}\right\|^{2} \leq C\left(1+\|h(\cdot, t)\|^{2}\right) .
$$

According to the Gronwall inequality, we have

$$
W^{\eta}\left(\xi_{u}(t)\right) \leq W^{\eta}\left(\xi_{u}(\tau)\right) \mathrm{e}^{-\delta(t-\tau)}+C\left(1+\int_{\tau}^{t}\|h(\cdot, s)\|^{2} \mathrm{~d} s\right) .
$$

Then, (23), (26), and $H_{0}^{1}(\Omega)^{\circ} L^{p+1}(\Omega)$ yield that

$$
\begin{aligned}
\left\|\xi_{u}(t)\right\|_{\mathscr{H}}^{2} \leq & \frac{1}{\kappa}\left(W^{\eta}\left(\xi_{u}(\tau)\right) \mathrm{e}^{-\delta(t-\tau)}+C\left(1+\int_{\tau}^{t}\|h(\cdot, s)\|^{2} \mathrm{~d} s\right)\right) \\
\leq & \left(\frac{\kappa_{1}}{\kappa}\left\|\xi_{u}(\tau)\right\|_{\mathscr{H}}^{2}+\frac{1}{\kappa} \Phi\left(\|\nabla u\|^{2}\right)+\frac{C_{3}}{\kappa}\|u\|_{L^{p+1}}^{p+1}+\frac{C_{4}}{\kappa}\right) \mathrm{e}^{-\delta(t-\tau)} \\
& +C\left(1+\int_{\tau}^{t}\|h(\cdot, s)\|^{2} \mathrm{~d} s\right) \leq C\left(\left\|\xi_{u}(\tau)\right\|_{\mathscr{C}}^{2}+\Phi\left(\|\nabla u\|^{2}\right)\right. \\
& \left.+\|\nabla u\|^{p+1}\right) \mathrm{e}^{-\delta(t-\tau)}+C\left(1+\int_{\tau}^{t}\|h(\cdot, s)\|^{2} \mathrm{~d} s\right) \\
\leq & C\left(\xi_{u}(\tau)_{\mathscr{H}}^{2}+\Phi\left(\left\|\xi_{u}(\tau)\right\|_{\mathscr{C}}^{2}\right)+\left(\left\|\xi_{u}(\tau)\right\|_{\mathscr{C}}^{2}\right)^{(p+1) / 2}\right) \mathrm{e}^{-\delta(t-\tau)} \\
& +C\left(1+\int_{\tau}^{t}\|h(\cdot, s)\|^{2} \mathrm{~d} s\right) \triangleq Q\left(\left\|\xi_{u}(\tau)\right\|_{\mathscr{H}}^{2}\right) \mathrm{e}^{-\delta(t-\tau)} \\
& +C\left(1+\int_{\tau}^{t}\|h(\cdot, s)\|^{2} \mathrm{~d} s\right),
\end{aligned}
$$


where $Q(x)=C\left(x+\Phi(x)+x^{(p+1) / 2}\right)>0$ is a monotone positive function on $\mathbb{R}^{+}$. Let

$$
\begin{aligned}
D_{0}(t) & =\left\{\xi \in \mathscr{H} \mid\|\xi\|_{\mathscr{H}} \leq R(t)\right\}, \text { with } R^{2}(t) \\
& =2 C\left(1+\|h\|_{L^{2}\left(-\infty, t ; L^{2}\right)}^{2}\right), \quad t \in \mathbb{R} .
\end{aligned}
$$

Obviously, $\mathscr{D}_{0}=\left\{D_{0}(t)\right\}_{t \in \mathbb{R}}$ is a pullback absorbing family of the process $U(t, \tau)$ in $\mathscr{H}$. Moreover, for every $t \in \mathbb{R}$, there exists a $T_{t}>0$ such that

$$
\begin{aligned}
& U(t, t-\tau) D_{0}(t-\tau) \subset D_{0}(t), \\
& \quad U(t-1, t-\tau) D_{0}(t-\tau) \subset D_{0}(t-1), \quad \text { for } \tau \geq T_{t} .
\end{aligned}
$$

Let $D(t)=\bigcup_{\tau \geq T_{t}} U(\vec{t}, t \tau) D_{0}(t \tau)^{\mathscr{H}}$. By a standard procedure (see, e.g., Theorem 3.1 of [34]), we know that $\mathscr{D}=$ $\{D(t)\}_{t \in \mathbb{R}}$ is a pullback absorbing family. Moreover, $D(t)$ is bounded in $\mathscr{H}_{1}$ for every $t \in \mathbb{R}$, and there exists a $T_{t}>0$ such that $U(t, t-\tau) D(t-\tau) \subset D(t)$ for $\tau \geq T_{t}$.

For simplicity, we assume that $\alpha>0$ and $L_{1}=L_{2}=1$ in the following.

Theorem 12. Let Assumptions 6-8 be in force. Then, the process $U(\cdot, \cdot)$ possesses a pullback attractor $\mathscr{A}=\{A(t)\}_{t \in \mathbb{R}}$ as shown in (4). Moreover, $A(t)$ is bounded in $\mathscr{H}_{1}$ for every $t$ $\in \mathbb{R}$.

Proof. According to Lemma 4, Theorem 5, Lemma 11, and the continuity of $U(t, \tau): \mathscr{H} \longrightarrow \mathscr{H}$, it suffices to show that $U(t, \tau)$ satisfies the condition (D-PC). Let $\left\{e_{j}\right\}_{j=1}^{\infty}$ be an orthonormal basis and $\left\{\lambda_{j}\right\}_{j=1}^{\infty}$ be the corresponding eigenvalues of $L^{2}(\Omega)$ which consists of eigenvectors of $-\Delta$, i.e., $\Delta e_{j}=\lambda_{j} e_{j}, j \in \mathbb{N}$. Let $V_{m} \times W_{m}=\operatorname{span}\left\{e_{1}, \cdots, e_{m}\right\} \times \operatorname{span}\left\{e_{1}\right.$, $\left.\cdots, e_{m}\right\}$ in $\mathscr{H}$ and $P_{m}=\left(P_{m}^{1}, P_{m}^{2}\right): \mathscr{H} \longrightarrow V_{m} \times W_{m}$ be an orthogonal projector. Denote $Q_{m}=I-P_{m}, u=P_{m}^{1} u+Q_{m}^{1} u=$ $u^{1}+u^{2}$, and $\xi_{u}^{\tau}(t)=\left(u(t), u_{t}(t)\right)=U(t, \tau)\left(u_{\tau}^{0}, u_{\tau}^{1}\right)$ with $\left(u_{\tau}^{0}\right.$, $\left.u_{\tau}^{1}\right) \in D(\tau), t \geq \tau$.

Let $\varepsilon>0$ and $t_{0} \in \mathbb{R}$ be given. Without loss of generality, we assume $\varepsilon<1 / 4$.

For every $\tau \geq 1$ and every $\left(u_{t_{0}-\tau}^{0}, u_{t_{0}-\tau}^{1}\right) \in D\left(t_{0}-\tau\right)$, let

$$
\begin{aligned}
\left(u, u_{t}\right)(t)= & \xi_{u}^{t_{0}-\tau}(t)=U\left(t, t_{0}-\tau\right)\left(u_{t_{0}-\tau}^{0}, u_{t_{0}-\tau}^{1}\right) \in U\left(t, t_{0}-\tau\right) \\
& \cdot D\left(t_{0}-\tau\right) \subset U\left(t, t_{0}-\tau\right) D_{0}\left(t_{0}-\tau\right) .
\end{aligned}
$$

Denote $Z(t)=(1 / 2)\left(\left\|u_{t}\right\|^{2}+\|u\|^{2}+\|\nabla u\|^{2}\right)$. It is easy to see that

$$
\begin{aligned}
Z\left(t_{0}-\tau+1\right) \leq & \frac{1}{2}\left(1+\frac{1}{\lambda_{1}}\right)\left\|\xi_{u}^{t_{0}-\tau}\left(t_{0}-\tau+1\right)\right\|_{\mathscr{H}}^{2} \\
\leq & \frac{1}{2}\left(1+\frac{1}{\lambda_{1}}\right) Q\left(\left\|\xi_{u}^{t_{0}-\tau}\left(t_{0}-\tau\right)\right\|_{\mathscr{H}}^{2} \cdot \mathrm{e}^{-\delta}\right. \\
& \left.+C\left(1+\int_{-\infty}^{t_{0}-\tau+1}\|h(\cdot, s)\|^{2} \mathrm{~d} s\right)\right) .
\end{aligned}
$$

we find

Since $\xi_{u}^{t_{0}-\tau}\left(t_{0}-\tau\right)=\left(u_{t_{0}-\tau}^{0}, u_{t_{0}-\tau}^{1}\right) \in D\left(t_{0}-\tau\right) \subset D_{0}\left(t_{0}-\tau\right)$,

$$
\left\|\xi_{u}^{t_{0}-\tau}\left(t_{0}-\tau\right)\right\|_{\mathscr{C}} \leq R\left(t_{0}-\tau\right) \leq R\left(t_{0}\right), \quad \forall \tau \geq 0 .
$$

Thus,

$$
\begin{aligned}
Z\left(t_{0}-\tau+1\right) & \leq \frac{1}{2}\left(1+\frac{1}{\lambda_{1}}\right)\left(Q\left(R^{2}\left(t_{0}\right)\right) \mathrm{e}^{-\delta}+C\left(1+\int_{-\infty}^{t_{0}}\|h(\cdot, s)\|^{2} \mathrm{~d} s\right)\right) \\
& \triangleq C_{5}\left(t_{0}\right), \quad \forall \tau \geq 1,
\end{aligned}
$$

where $C_{5}\left(t_{0}\right)$ is independent of $\tau$. Then, there exists $\tau_{0}>1$ such that

$$
Z\left(t_{0}-\left(\tau_{0}-1\right)\right) \mathrm{e}^{-2 \varepsilon^{2 \alpha}\left(\tau_{0}-1\right)}<\frac{\varepsilon^{2}}{2} .
$$

On the other hand, for every $\left(u_{t_{0}-\tau_{0}}^{0}, u_{t_{0}-\tau_{0}}^{1}\right) \in D\left(t_{0}-\tau_{0}\right)$, using (16) and (18), we get that

$$
\|\nabla u(t)\|^{2}+\left\|\nabla u_{t}(t)\right\|^{2}+\left\|u_{t t}(t)\right\|_{H^{-1}}^{2} \leq K_{0}, \quad \text { for } t \in\left[t_{0}-\tau_{0}+1, t_{0}\right],
$$

where $\left(u(t), u_{t}(t)\right)=U\left(t, t_{0}-\tau_{0}\right)\left(u_{t_{0}-\tau_{0}}^{0}, u_{t_{0}-\tau_{0}}^{1}\right)$. Using $H_{0}^{1}(\Omega)^{\circ} L^{q}(\Omega)\left(2 \leq q \leq p^{*}=2 n /(n-2)\right)$, one can find $M \geq 1$, $L_{0}<t_{0}$ (without loss of generality, we assume $L_{0}<0$ ), such that for every $t \in\left[t_{0}-\tau_{0}+1, t_{0}\right]$,

$$
\begin{aligned}
& \|f(u(t))\|_{L^{(p+1) / p}}+\left(\int_{t}^{t+1}\left\|u_{t t}(s)\right\|_{H^{-1}}^{2} \mathrm{~d} s\right)^{1 / 2}+\left(\int_{-\infty}^{t_{0}}\|h(\cdot, s)\|^{2} \mathrm{~d} s\right)^{1 / 2} \\
& <M, \int_{-\infty}^{L_{0}}\|h(\cdot, s)\|^{2} \mathrm{~d} s<\frac{\varepsilon^{2}}{4} .
\end{aligned}
$$

By the Sobolev embedding theorem, we know that the embedding $H_{0}^{1}(\Omega)^{\circ} L^{2}(\Omega)$ is compact. Then, the boundedness of $\left\{u(t), u_{t}(t)\right\}_{t \in\left[t_{0}-\tau_{0}+1, t_{0}\right]}$ in $H_{0}^{1}(\Omega) \times H_{0}^{1}(\Omega)$ implies that $\left\{u(t), u_{t}(t)\right\}_{t \in\left[t_{0}-\tau_{0}+1, t_{0}\right]}$ is compact in $L^{2}(\Omega) \times L^{2}(\Omega)$. Therefore, for $\varepsilon_{1}=\varepsilon^{2+2 \alpha} / 4 M\left(1+\sqrt{t_{0}-L_{0}}\right)$, there exists $m_{0} \in \mathbb{Z}_{+}$, such that for every $t \in\left[t_{0}-\tau_{0}+1, t_{0}\right]$,

$$
\begin{gathered}
\left\|\left(u^{2}(t), u_{t}^{2}(t)\right)\right\|_{L^{2} \times L^{2}}<\varepsilon_{1}, \\
\left\|u^{2}\right\|_{L^{p^{+1}}} \leq\left\|u^{2}\right\|^{\theta} \cdot\left\|u^{2}\right\|_{L^{p^{*}}}^{1-\theta} \leq C\left\|u^{2}\right\|^{\theta} \cdot\left\|\nabla u^{2}\right\|^{1-\theta} \leq C_{6}\left\|u^{2}\right\|^{\theta}<\frac{\varepsilon_{1}^{2}}{M},
\end{gathered}
$$


where $u=P_{m_{0}}^{1} u+\left(I-P_{m_{0}}^{1}\right) u \triangleq u^{1}+u^{2}, u_{t}=P_{m_{0}}^{2} u_{t}+(I-$ $\left.P_{m_{0}}^{2}\right) u_{t} \triangleq u_{t}^{1}+u_{t}^{2}$, and $1 /(p+1)=\theta / 2+(1-\theta) / p^{*}$.

Now, we will consider two situations. Without loss of generality, we assume $0<\varepsilon<1 / 3$.

Case 1 . For every $\left(u_{t_{0}-\tau_{0}}^{0}, u_{t_{0}-\tau_{0}}^{1}\right) \in D\left(t_{0}-\tau_{0}\right)$, the inequality

$$
\|\nabla u(t)\|>\varepsilon,
$$

holds for any $t \in\left[t_{0}-\tau_{0}+1, t_{0}\right]$, where $\left(u, u_{t}\right)(t)=\xi_{u}^{t_{0}-\tau_{0}}(t)$.

Multiplying (1) by $u^{2}$, we have that

$$
\begin{aligned}
& \frac{d}{d t}\left(\left(u_{t}^{2}, u^{2}\right)+\frac{1}{2}\left\|\nabla u^{2}\right\|^{2}\right)+\phi\left(\|\nabla u(t)\|^{2}\right)\left\|\nabla u^{2}\right\|^{2} \\
& \quad \leq\left\|u_{t}^{2}\right\|^{2}+\left(f(u), u^{2}\right)+\left(h(\cdot, t), u^{2}\right) .
\end{aligned}
$$

Let $Y(t)=\left(u_{t}^{2}, u^{2}\right)+(1 / 2)\left\|\nabla u^{2}\right\|^{2}$. Since $\phi\left(\|\nabla u\|^{2}\right) \geq$ $\min \left\{\|\nabla u\|^{2 \alpha}, 1\right\} \geq \min \left\{\varepsilon^{2 \alpha}, 1\right\}=\varepsilon^{2 \alpha}$ in this case, the above inequality implies that

$$
\begin{aligned}
\frac{d}{d t} Y(t) & +2 \varepsilon^{2 \alpha} Y(t) \leq 2 \varepsilon^{2 \alpha}\left(u_{t}^{2}, u^{2}\right)+\left\|u_{t}^{2}\right\|^{2}+\left(f(u), u^{2}\right) \\
& +\left(h(\cdot, t), u^{2}\right) \leq 2 \varepsilon^{2 \alpha} \cdot\left\|u_{t}^{2}\right\| \cdot\left\|u^{2}\right\|+\left\|u_{t}^{2}\right\|^{2} \\
& +\|f(u)\|_{L^{(p+1) / p}} \cdot\left\|u^{2}\right\|_{L^{p+1}}+\|h(\cdot, t)\| \cdot\left\|u^{2}\right\| \triangleq W^{\varepsilon}(t) .
\end{aligned}
$$

By Gronwall's inequality, we obtain that

$$
Y(t) \leq Y(t-(\tau-1)) \mathrm{e}^{-2 \varepsilon^{2 \alpha}(\tau-1)}+\mathrm{e}^{-2 \varepsilon^{2 \alpha} t} \int_{t-(\tau-1)}^{t} \mathrm{e}^{2 \varepsilon^{2 \alpha} s} W^{\varepsilon}(s) \mathrm{d} s .
$$

Since

$$
Y\left(t_{0}-\tau+1\right) \leq Z\left(t_{0}-\tau+1\right) \leq C_{5}\left(t_{0}\right),
$$

(37) yields that

$$
Y\left(t_{0}-\left(\tau_{0}-1\right)\right) \mathrm{e}^{-2 \varepsilon^{2 \alpha}\left(\tau_{0}-1\right)}<\frac{\varepsilon^{2}}{2} .
$$

Combining (45), we have

$$
\begin{aligned}
Y\left(t_{0}\right) \leq & Y\left(t_{0}-\tau_{0}+1\right) \mathrm{e}^{-2 \varepsilon^{2 \alpha}\left(\tau_{0}-1\right)}+\mathrm{e}^{-2 \varepsilon^{2 \alpha} t_{0}} \int_{t_{0}-\tau_{0}+1}^{t_{0}} \mathrm{e}^{2 \varepsilon^{2 \alpha} s} W^{\varepsilon}(s) \mathrm{d} s \\
\leq & \frac{\varepsilon^{2}}{2}+\mathrm{e}^{-2 \varepsilon^{2 \alpha} t_{0}} \int_{t_{0}-\tau_{0}+1}^{t_{0}} \mathrm{e}^{2 \varepsilon^{2 \alpha} s} 2 \varepsilon_{1}^{2}\left(\varepsilon^{2 \alpha}+1\right) \mathrm{d} s \\
& +\mathrm{e}^{-2 \varepsilon^{2 \alpha} t_{0}} \int_{t_{0}-\tau_{0}+1}^{t_{0}} \mathrm{e}^{2 \varepsilon^{2 \alpha} s} \cdot\|h(\cdot, s)\| \cdot\left\|u^{2}(s)\right\| \mathrm{d} s \\
\leq & \frac{\varepsilon^{2}}{2}+\left(1+\frac{1}{\varepsilon^{2 \alpha}}\right) \varepsilon_{1}^{2}+\mathrm{e}^{-2 \varepsilon^{2 \alpha} t_{0}} \int_{t_{0}-\tau_{0}+1}^{t_{0}} \mathrm{e}^{2 \varepsilon^{2 \alpha} s} \cdot\|h(\cdot, s)\| \cdot\left\|u^{2}(s)\right\| \mathrm{d} s .
\end{aligned}
$$

If $L_{0} \leq t_{0}-\tau_{0}+1$, by the Hölder inequality, we have that

$$
\begin{aligned}
I_{1} & =\mathrm{e}^{-2 \varepsilon^{2 \alpha} t_{0}} \int_{t_{0}-\tau_{0}+1}^{t_{0}} \mathrm{e}^{2 \varepsilon^{2 \alpha} s} \cdot\|h(\cdot, s)\| \cdot\left\|u^{2}(s)\right\| \mathrm{d} s \\
& \leq \mathrm{e}^{-2 \varepsilon^{2 \alpha} t_{0}} \cdot \mathrm{e}^{2 \varepsilon^{2 \alpha} t_{0}} \varepsilon_{1} \cdot \sqrt{t_{0}-L_{0}} \cdot M<\frac{\varepsilon^{2}}{4} .
\end{aligned}
$$

On the other hand, if $L_{0}>t_{0}-\tau_{0}+1$, we get that

$$
\begin{aligned}
I_{1} \leq & \mathrm{e}^{-2 \varepsilon^{2 \alpha} t_{0}}\left[\int_{L_{0}}^{t_{0}} \mathrm{e}^{2 \varepsilon^{2 \alpha} t_{0}} \cdot\|h(\cdot, s)\| \cdot \varepsilon_{1} \mathrm{~d} s+\frac{1}{2} \int_{t_{0}-\tau_{0}+1}^{L_{0}}\right. \\
& \left.\cdot \mathrm{e}^{2 \varepsilon^{2 \alpha} s}\left(\left\|u^{2}(s)\right\|^{2}+\|h(\cdot, s)\|^{2}\right) \mathrm{d} s\right] \\
< & \varepsilon_{1} \cdot \sqrt{t_{0}-L_{0}} \cdot M+\frac{\varepsilon_{1}^{2}}{4 \varepsilon^{2 \alpha}}+\frac{\varepsilon^{2}}{8}<\frac{\varepsilon^{2}}{2} .
\end{aligned}
$$
because

The above inequalities guarantee that $Y\left(t_{0}\right)<9 \varepsilon^{2} / 8$. And

$$
\begin{aligned}
Y\left(t_{0}\right)= & \left(u_{t}^{2}\left(t_{0}\right), u^{2}\left(t_{0}\right)\right)+\frac{1}{2}\left\|\nabla u^{2}\left(t_{0}\right)\right\|^{2} \geq \frac{1}{2}\left\|\nabla u^{2}\left(t_{0}\right)\right\|^{2} \\
& -\left\|u_{t}^{2}\left(t_{0}\right)\right\| \cdot\left\|u^{2}\left(t_{0}\right)\right\| \geq \frac{1}{2}\left\|\nabla u^{2}\left(t_{0}\right)\right\|^{2}-\varepsilon_{1}^{2},
\end{aligned}
$$

we get that

$$
\left\|\nabla u^{2}\left(t_{0}\right)\right\|^{2} \leq 2\left(Y\left(t_{0}\right)+\varepsilon_{1}^{2}\right)<2\left(\frac{9 \varepsilon^{2}}{8}+\frac{\varepsilon^{2}}{8}\right)<4 \varepsilon^{2},
$$

$$
\text { i.e., }\left\|\nabla u^{2}\left(t_{0}\right)\right\|<2 \varepsilon \text {. }
$$

Case 2. There exist $\left(u_{t_{0}-\tau_{0}}^{0}, u_{t_{0}-\tau_{0}}^{1}\right) \in D\left(t_{0}-\tau_{0}\right)$ and $t_{1} \in\left[t_{0}-\right.$ $\left.\tau_{0}+1, t_{0}\right]$ such that

$$
\left\|\nabla u\left(t_{1}\right)\right\| \leq \varepsilon \text { with }\left(u, u_{t}\right)(t)=\xi_{u}^{t_{0}-\tau}(t) .
$$

In this case, we claim that the following inequality is true, i.e., for every $t_{1} \leq t \leq t_{0}$,

$$
\left\|\nabla u^{2}(t)\right\|<2 \varepsilon, \quad \text { for } u^{2}=Q_{m_{0}}^{1} u .
$$

In fact, if this claim is not true, the continuity of $\| \nabla$ $u^{2}(t) \|$ gives that

$$
E=\left\{t \mid t \in\left[t_{1}, t_{0}\right],\left\|\nabla u^{2}(t)\right\|=2 \varepsilon\right\},
$$

is not an empty set. Let $t_{3}=\inf E$. It is easy to prove that $\left\|\nabla u^{2}\left(t_{3}\right)\right\|=2 \varepsilon$. Moreover, by the definition of $t_{3}$, we have that

$$
\left\|\nabla u^{2}(t)\right\|<2 \varepsilon, \quad \forall t \in\left[t_{1}, t_{3}\right) .
$$

According to the intermediate value theorem, we know that the set 


$$
E_{1}=\left\{t \mid t \in\left(t_{1}, t_{3}\right),\left\|\nabla u^{2}(t)\right\|=\frac{3}{2} \varepsilon\right\}
$$

is not empty. Denoting $t_{2}=\sup E_{1}$, we can conclude from the definition of supremum that

$$
\left\|\nabla u^{2}\left(t_{2}\right)\right\|=\frac{3}{2} \varepsilon .
$$

Thus,

$$
\begin{aligned}
\frac{3}{2} \varepsilon & <\left\|\nabla u^{2}(t)\right\|<3 \varepsilon, \forall t \in\left(t_{2}, t_{3}\right],\left\|\nabla u^{2}\left(t_{2}\right)\right\| \\
& =\frac{3}{2} \varepsilon,\left\|\nabla u^{2}\left(t_{3}\right)\right\|=2 \varepsilon .
\end{aligned}
$$

Notice that $\|\nabla u\| \geq\left\|\nabla u^{2}\right\|$ and $\left\|\nabla u^{2}\right\| \leq 1$ for $t \in\left[t_{2}, t_{3}\right]$; we have that $\phi\left(\|\nabla u\|^{2}\right) \geq\left\|\nabla u^{2}\right\|^{2 \alpha}$. Then, integrating (43) on $\left(t_{2}, t_{3}\right)$, we have that

$$
\begin{aligned}
& \left(\left(u_{t}^{2}\left(t_{3}\right), u^{2}\left(t_{3}\right)\right)+\frac{1}{2}\left\|\nabla u^{2}\left(t_{3}\right)\right\|^{2}\right)-\left(\left(u_{t}^{2}\left(t_{2}\right), u^{2}\left(t_{2}\right)\right)+\frac{1}{2}\left\|\nabla u^{2}\left(t_{2}\right)\right\|^{2}\right) \\
& \quad+\int_{t_{2}}^{t_{3}} \phi\left(\|\nabla u(s)\|^{2}\right)\left\|\nabla u^{2}(s)\right\|^{2} \mathrm{~d} s \\
& \leq \int_{t_{2}}^{t_{3}}\left(\left\|u_{t}(s)^{2}\right\|^{2}+\left(f(u(s)), u^{2}(s)\right)+\left(h(\cdot, s), u^{2}(s)\right)\right) \mathrm{d} s .
\end{aligned}
$$

It implies that

$$
\begin{aligned}
& \left\|\nabla u^{2}\left(t_{3}\right)\right\|^{2}+2 \int_{t_{2}}^{t_{3}}\left\|\nabla u^{2}(s)\right\|^{2+2 \alpha} \mathrm{d} s \leq\left\|\nabla u^{2}\left(t_{2}\right)\right\|^{2} \\
& \quad-2\left(u_{t}^{2}\left(t_{3}\right), u^{2}\left(t_{3}\right)\right)+2\left(u_{t}^{2}\left(t_{2}\right), u^{2}\left(t_{2}\right)\right) \\
& \quad+2 \int_{t_{2}}^{t_{3}}\left(\left\|u_{t}(s)^{2}\right\|^{2}+\left(f(u(s)), u^{2}(s)\right)+\left(h(\cdot, s), u^{2}(s)\right)\right) \mathrm{d} s .
\end{aligned}
$$

Combing (40), (41), and (59),we get

$$
\begin{aligned}
& \left\|\nabla u^{2}\left(t_{3}\right)\right\|^{2}+2\left(\frac{3}{2} \varepsilon\right)^{2 \alpha+2}\left(t_{3}-t_{2}\right) \leq\left\|\nabla u^{2}\left(t_{3}\right)\right\|^{2} \\
& \quad+2 \int_{t_{2}}^{t_{3}}\left\|\nabla u^{2}(s)\right\|^{2 \alpha+2} \mathrm{~d} s \leq\left\|\nabla u^{2}\left(t_{2}\right)\right\|^{2}+2\left\|u_{t}^{2}\left(t_{2}\right)\right\| \\
& \quad+\left\|u^{2}\left(t_{2}\right)\right\|+2\left\|u_{t}^{2}\left(t_{3}\right)\right\| \cdot\left\|u^{2}\left(t_{3}\right)\right\| \\
& \quad+2 \int_{t_{2}}^{t_{3}}\left(\left\|u_{t}^{2}(s)\right\|^{2}+\left(f(u), u^{2}\right)+\left(h(\cdot, s), u^{2}\right)\right) \mathrm{d} s \\
& \leq\left(\frac{3}{2} \varepsilon\right)^{2}+4 \varepsilon_{1}^{2}+2 \varepsilon_{1}^{2}\left(t_{3}-t_{2}\right)+2 \int_{t_{2}}^{t_{3}}\|f(u)\|_{L^{(p+1) / p}}\|\| u^{2} \|_{L^{p+1}} \mathrm{~d} s \\
& \quad+2 \varepsilon_{1}\left(\int_{t_{2}}^{t_{3}}\|h(\cdot, s)\|^{2} \mathrm{~d} s\right)^{1 / 2} \sqrt{t_{3}-t_{2}} \leq\left(\frac{3}{2} \varepsilon\right)^{2}+4 \varepsilon_{1}^{2} \\
& \quad+2 \varepsilon_{1}^{2}\left(t_{3}-t_{2}\right)+2 \int_{t_{2}}^{t_{3}} M \cdot \frac{\varepsilon_{1}^{2}}{M} \mathrm{~d} s+2 \varepsilon_{1} M \sqrt{t_{3}-t_{2}} \leq\left(\frac{3}{2} \varepsilon\right)^{2} \\
& \quad+4 \varepsilon_{1}^{2}+4 \varepsilon_{1}^{2}\left(t_{3}-t_{2}\right)+\varepsilon_{1} M\left(t_{3}-t_{2}\right)+\varepsilon_{1} M \leq \frac{9}{4} \varepsilon^{2}+\frac{\varepsilon^{4+4 \alpha}}{4} \\
& \quad+\frac{\varepsilon^{4+4 \alpha}}{4}\left(t_{3}-t_{2}\right)+\frac{\varepsilon^{2+2 \alpha}}{4}\left(t_{3}-t_{2}\right)+\frac{\varepsilon^{2+2 \alpha}}{4}<\frac{11}{4} \varepsilon^{2}+\frac{1}{2} \varepsilon^{2+2 \alpha}\left(t_{3}-t_{2}\right) .
\end{aligned}
$$

Thus, $\left\|\nabla u^{2}\left(t_{3}\right)\right\|^{2}<(11 / 4) \varepsilon^{2}$, which is in contradiction with (59), and condition (D-PC) holds. This completes the proof.

\section{Appendix}

\section{A. Proof of Proposition 10}

We prove the well-posedness of Problem (1) using the same method as in [25].

Step 1. We start with the case when $u_{\tau}^{0} \in H^{2}(\Omega) \cap H_{0}^{1}(\Omega)$ and assume that $\left\|\left(u_{\tau}^{0}, u_{\tau}^{1}\right)\right\|_{\mathscr{H}} \leq R$ with some $R>0$. We seek for the approximate solutions of the form

$$
u^{N}(t)=\sum_{k=1}^{N} g_{k}(t) e_{k}, \quad N=1,2, \cdots
$$

satisfying the finite-dimensional projections of (1). Moreover, we have that

$$
\begin{aligned}
& \left\|\left(u^{N}(\tau), u_{t}^{N}(\tau)\right)\right\|_{\mathscr{H}} \leq C_{R}, \\
& \quad\left\|\left(u^{N}(\tau)-u_{\tau}^{0}, u_{t}^{N}(\tau)-u_{\tau}^{1}\right)\right\|_{\mathscr{H}} \longrightarrow 0, \text { as } N \longrightarrow \infty .
\end{aligned}
$$

We omit the superscript $N$ in the sequel. Now, we use the multiplier $u_{t}(t)$ and get that

$$
\frac{d}{d t}\left[\frac{1}{2}\left(\left\|u_{t}\right\|^{2}+\Phi\left(\|\nabla u\|^{2}\right)\right)+F(u(t))\right]+\left\|\nabla u_{t}\right\|^{2}-\left(h, u_{t}(t)\right)=0 .
$$

Similarly, multiplying (1) by $u$, we have that

$$
\begin{aligned}
\frac{d}{d t}\left[\left(u, u_{t}\right)+\frac{1}{2}\|\nabla u\|^{2}\right]= & \left\|u_{t}\right\|^{2}-\phi\left(\|\nabla u\|^{2}\right)\|\nabla u\|^{2} \\
& -(f(u), u)+(h, u) .
\end{aligned}
$$

Let

$$
\begin{gathered}
E\left(u_{0}, u_{1}\right)=\frac{1}{2}\left(\left\|u_{1}\right\|^{2}+\Phi\left(\left\|\nabla u_{0}\right\|^{2}\right)\right)+F\left(u_{0}\right), \\
W^{\eta}\left(u_{0}, u_{1}\right)=E\left(u_{0}, u_{1}\right)+\eta\left[\left(u_{0}, u_{1}\right)+\frac{1}{2}\left\|\nabla u_{0}\right\|^{2}\right] .
\end{gathered}
$$

From (A3) and (A4),

$$
\begin{aligned}
\frac{d}{d t} W^{\eta}(u(t) & \left., u_{t}(t)\right)+\left\|\nabla u_{t}\right\|^{2}-\left(h, u_{t}(t)\right) \\
= & \eta\left(\left\|u_{t}\right\|^{2}-\phi\left(\|\nabla u\|^{2}\right)\|\nabla u\|^{2}-(f(u), u)+(h, u)\right) .
\end{aligned}
$$

Using (14), (24), and $\left|\left(h, u_{t}(t)\right)\right| \leq \lambda_{1} / 2\left\|u_{t}\right\|^{2}+1 / 2 \lambda_{1}$ $\|h(\cdot, t)\|^{2}$, we find that 


$$
\begin{gathered}
\frac{d}{d t} W^{\eta}\left(u(t), u_{t}(t)\right)+\frac{1}{2}\left\|\nabla u_{t}\right\|^{2} \leq \eta\left\|u_{t}\right\|^{2}-\eta \delta_{0} \Phi\left(\|\nabla u\|^{2}\right) \\
+\eta \theta_{1}\|u\|^{2}+c_{1}\|h(\cdot, t)\|^{2}+c_{2} .
\end{gathered}
$$

where $c_{1}, c_{2}$ is independent of $t$. Obviously,

$$
W^{\eta}\left(u_{0}, u_{1}\right) \leq\left\|u_{1}\right\|^{2}+\frac{1}{2} \Phi\left(\left\|\nabla u_{0}\right\|^{2}\right)+\tilde{c}_{0}\left\|\nabla u_{0}\right\|^{2} .
$$

By (13) and (14), there exists $\eta_{0}>, \delta_{1}>0$, for any $\eta$ $\epsilon\left(0, \eta_{0}\right)$,

$$
\begin{aligned}
W^{\eta}\left(u_{0}, u_{1}\right) \geq & \left(\frac{1}{2}-\eta\right)\left\|u_{1}\right\|^{2}+\frac{1}{2} \phi_{1} \cdot\left\|\nabla u_{0}\right\|^{2}-\frac{\theta_{1}}{2}\left\|u_{0}\right\|^{2} \\
& -\eta\left\|u_{0}\right\|^{2}-\frac{\eta}{2}\left\|\nabla u_{0}\right\|^{2}-\tilde{c}_{1} \geq \frac{1}{4}\left\|u_{1}\right\|^{2} \\
& +\delta_{1}\left\|\nabla u_{0}\right\|^{2}-\tilde{c}_{2} .
\end{aligned}
$$

Combing (A8) and the above inequalities, we have that

$$
\begin{gathered}
\frac{d}{d t} W^{\eta}\left(u(t), u_{t}(t)\right)+\frac{1}{2}\left\|\nabla u_{t}\right\|^{2} \leq C_{1} W^{\eta}\left(u(t), u_{t}(t)\right) \\
+C_{2}\|h(\cdot, t)\|^{2}+C_{3} .
\end{gathered}
$$

Therefore, using Gronwall's inequality, we obtain

$W^{\eta}\left(u(t), u_{t}(t)\right) \leq \tilde{C}_{R, T}+C_{2} e^{C_{1} T} \int_{-\infty}^{T}\|h(\cdot s)\|^{2} \mathrm{~d} s \triangleq C_{R, T}^{1}, \quad \forall t \in[\tau, T]$,

which means that

$$
\begin{gathered}
\left\|\left(u(t), u_{t}(t)\right)\right\|_{\mathscr{H}} \leq C_{R, T}, \quad \forall t \in[\tau, T], \\
\int_{\tau}^{T}\left\|\nabla u_{t}(t)\right\|^{2} d t \leq C_{R, T} .
\end{gathered}
$$

Now, multiplying (1) by $-\Delta u$, we have

$$
\begin{aligned}
\frac{d}{d t}[- & \left.\left(u_{t}, \Delta u\right)+\frac{1}{2}\|\Delta u\|^{2}\right]+\phi\left(\|\nabla u(t)\|^{2}\right)\|\Delta u\|^{2} \\
& +\left(f^{\prime}(u),|\nabla u|^{2}\right) \leq\left\|\nabla u_{t}(t)\right\|^{2}+\frac{1}{2}\|h(\cdot, t)\|^{2}+\frac{1}{2}\|\Delta u\|^{2} .
\end{aligned}
$$

Since $H_{0}^{1}(\Omega)^{\circ} L^{p+1}(\Omega)$ when $n \geq 3$ and $H_{0}^{1}(\Omega)^{\circ} L^{q}(\Omega)$ for any $q \geq 1$ when $n=2, H_{0}^{1}(\Omega)^{\circ} L^{\infty}(\Omega)$ when $n=1$, we easily obtain that

$$
\begin{aligned}
\left|\left(f^{\prime}(u),|\nabla u|^{2}\right)\right| \leq & C \int_{\Omega}\left(1+|u|^{p-1}\right)|\nabla u|^{2} d x \leq C\|\nabla u\|^{2} \\
& +C\|u\|_{p+1}^{p-1} \cdot\|\nabla u\|_{p+1}^{2} \leq C\|\nabla u\|^{2} \\
& +\|\nabla u\|^{p-1} \cdot\|\Delta u\|^{2} \leq C\left(1+\|\Delta u\|^{2}\right) .
\end{aligned}
$$

It follows that

$$
\frac{d}{d t}\left[-\left(u_{t}, \Delta u\right)+\frac{1}{2}\|\Delta u\|^{2}\right] \leq\left\|\nabla u_{t}(t)\right\|^{2}+\frac{1}{2}\|h(\cdot, t)\|^{2}+C_{1}\|\Delta u\|^{2}+C_{2},
$$

for every $t \in[\tau, T]$. Let

$$
\Psi(t)=E\left(u(t), u_{t}(t)\right)+\varepsilon\left[-\left(u_{t}, \Delta u\right)+\frac{1}{2}\|\Delta u\|^{2}\right], \quad \varepsilon>0 .
$$

We can choose $\varepsilon_{0}>0$, such that

$$
\Psi(t) \geq C_{R, T, \varepsilon}\left(\left\|u_{t}\right\|^{2}+\|\Delta u\|^{2}\right)-C, \quad \forall 0<\varepsilon<\varepsilon_{0} .
$$

Thus, combing (A3), (A12), and (A15), we have that $\frac{d}{d t} \Psi(t) \leq C_{1}\|\Delta u\|^{2}+C_{2}\|h(\cdot, t)\|^{2}+C_{3} \leq C_{4} \Psi(t)+C_{2}\|h(\cdot, t)\|^{2}+C_{5}$.

This implies that

$$
\|\Delta u(t)\|^{2} \leq C_{R, T}\left(1+\|\Delta u(\tau)\|^{2}\right), \quad t \in[\tau, T] .
$$

The above a priori estimates show that $\left(u^{N}, u_{t}^{N}\right)$ is bounded in

$$
L^{\infty}\left(\tau, T ; H^{2}(\Omega) \cap H_{0}^{1}(\Omega)\right) \times\left[L^{\infty}\left(0, T ; L^{2}(\Omega)\right) \cap L^{2}\left(\tau, T ; H_{0}^{1}(\Omega)\right)\right],
$$

for every $T>\tau$. Moreover, using the equation for $u^{N}(t)$, we can show $\int_{\tau}^{T}\left\|u_{t t}^{N}\right\|_{-m}^{2} \mathrm{~d} t \leq C_{R, T}$ for some $m \geq \max \{1, n /$ $2\}$. Thus, there exists a subsequence, stilled denoted $u^{N}$, and $u$, such that

$$
\begin{gathered}
u^{N} \longrightarrow u, \quad \text { in } C\left(\tau, T ; H_{0}^{1}(\Omega)\right), \\
u^{N} \longrightarrow u, \quad \text { in } L^{\infty}\left(\tau, T ; H^{2}(\Omega)\right) \text { weak-star, } \\
u_{t}^{N} \longrightarrow u_{t}, \quad \operatorname{in} L^{2}\left(\tau, T ; L^{2}(\Omega)\right) \cap C\left(\tau, T ; H^{-1}(\Omega)\right), \\
u_{t}^{N} \longrightarrow u_{t}, \quad \text { in } L^{2}\left(\tau, T ; H_{0}^{1}(\Omega)\right) \text { weak, }
\end{gathered}
$$

as $N \longrightarrow \infty$. Moreover, by the Lions lemma (see Lemma 1.3 in [37]) we have that 


$$
f\left(u^{N}(x, t)\right) \longrightarrow f(u(x, t)), \quad \text { in } L^{2}([\tau, T] \times \Omega) \text { weak },
$$

as $N \longrightarrow \infty$. Then, making a limit transition in the nonlinear term, we prove the existence of a weak solution under the additional condition $u_{\tau}^{0} \in H^{2}(\Omega) \cap H_{0}^{1}(\Omega)$. One can see that this solution $u$ satisfies (14) and (15).

Step 2. Now, let $u^{1}(t)$ and $u^{2}(t)$ be weak solutions to (1) with different initial data $\left(u_{0}^{i}, u_{1}^{i}\right) \in \mathscr{H}$ such that

$$
\left\|\left(u^{i}(t), u_{t}^{i}(t)\right)\right\|_{\mathscr{H}}+\int_{\tau}^{T}\left\|\nabla u_{t}(t)\right\|^{2} \mathrm{~d} t \leq C_{R}, \quad \forall t \in[\tau, T],
$$

for some $R>0$. Notice that we do not assume $u_{0}^{i} \epsilon$ $H^{2}(\Omega)$ here. Since $\phi \in C^{1}$, we conclude from (60) that

$$
\left|\phi\left(\|\nabla u\|^{2}\right)\right|,\left|\phi^{\prime}\left(\|\nabla u\|^{2}\right)\right| \leq M, t \in[\tau, T] .
$$

We can see that $z(t)=u^{1}(t)-u^{2}(t)$ solves the equation

$$
\begin{aligned}
z_{t t}-\Delta z_{t}-\frac{1}{2} \phi_{12}(t) \Delta z- & \frac{1}{2}\left[\phi_{1}(t)-\phi_{2}(t)\right]\left(\Delta u^{1}+\Delta u^{2}\right) \\
+ & f\left(u^{1}\right)-f\left(u^{2}\right)=0
\end{aligned}
$$

where $\phi_{12}(t)=\phi_{1}(t)+\phi_{2}(t)$ with $\phi_{i}(t)=\phi\left(\left\|\nabla u^{i}(t)\right\|^{2}\right)$. By the definition of a weak solution, we can multiply (A25) by $z$ in $L^{2}(\Omega)$ and reduce that

$$
\begin{aligned}
& \frac{d}{d t}\left[\left(z, z_{t}\right)+\frac{1}{2}\|\nabla z\|^{2}\right]-\left\|z_{t}\right\|^{2}+\frac{1}{2} \phi_{12}(t)\|\nabla z\|^{2} \\
& \quad+\left(f\left(u^{1}\right)-f\left(u^{2}\right), z\right)+\frac{1}{2}\left[\phi_{1}(t)-\phi_{2}(t)\right]\left(\nabla u^{1}+\nabla u^{2}, \nabla z\right)=0 .
\end{aligned}
$$

Using $H_{0}^{1}(\Omega) \hookrightarrow L^{q}(\Omega)$ for every $1 \leq q<+\infty$ when $n=$ 1,2 and $H_{0}^{1}(\Omega) \hookrightarrow L^{2 n /(n-2)}(\Omega)$ when $n \geq 3$, we have that

$$
\begin{aligned}
\left|\left(f\left(u^{1}\right)-f\left(u^{2}\right), z\right)\right| & \leq C \int_{\Omega}\left(1+\left|u^{1}\right|^{p-1}+\left|u^{2}\right|^{p-1}\right)|z|^{2} d x \\
& \leq C_{R}\|\nabla z\|^{2} .
\end{aligned}
$$

Therefore, combining with

$$
\begin{aligned}
\left|\phi_{1}(t)-\phi_{2}(t)\right|= & \mid \int_{0}^{1} \phi^{\prime}\left(\lambda\left\|\nabla u^{1}(t)\right\|^{2}+(1-\lambda)\left\|\nabla u^{2}(t)\right\|^{2}\right) d \lambda \\
& \cdot\left(\nabla\left(u^{1}+u^{2}\right), \nabla z\right) \mid \leq C\|\nabla z\|,
\end{aligned}
$$

we can conclude that

$$
\frac{d}{d t}\left[\left(z, z_{t}\right)+\frac{1}{2}\|\nabla z\|^{2}\right] \leq\left\|z_{t}\right\|^{2}+C_{R}\|\nabla z\|^{2} .
$$

Now consider the multiplier $z_{t}$. Since $z \in L^{\infty}\left(\tau, T ; H_{0}^{1}\right.$ $(\Omega)), z_{t} \in L^{2}\left(\tau, T ; H_{0}^{1}(\Omega)\right)$, and $z_{t t} \in L^{2}\left(\tau, T ; H^{-1}(\Omega)\right)$, we can multiply (A26) by $z_{t}$ and obtain

$$
\begin{aligned}
\frac{1}{2} \frac{d}{d t}\left\|z_{t}\right\|^{2} & +\left\|\nabla z_{t}\right\|^{2}+\frac{1}{2} \phi_{12}(t)\left(\nabla z, \nabla z_{t}\right)+\left(f\left(u^{1}\right)-f\left(u^{2}\right), z_{t}\right) \\
& -\frac{1}{2}\left[\phi_{1}(t)-\phi_{2}(t)\right]\left(\Delta\left(u^{1}+u^{2}\right), z_{t}\right)=0 .
\end{aligned}
$$

Similar to (A29), we can get

$$
\frac{d}{d t}\left\|z_{t}\right\|^{2}+2\left\|\nabla z_{t}\right\|^{2}+2\left(f\left(u^{1}\right)-f\left(u^{2}\right), z_{t}\right) \leq C\|\nabla z\| \cdot\left\|\nabla z_{t}\right\| .
$$

Similar to (A27), we have

$$
\left|\left(f\left(u^{1}\right)-\mathrm{f}\left(u^{2}\right), z_{t}\right)\right| \leq C_{R}\|\nabla z\| \cdot\left\|\nabla z_{t}\right\| .
$$

Therefore, we can conclude from Young's inequality that

$$
\frac{d}{d t}\left\|z_{t}\right\|^{2}+\left\|\nabla z_{t}\right\|^{2} \leq C\left(\|\nabla z\|^{2}+\left\|\nabla z_{t}\right\|^{2}\right)
$$

Let

$$
\Gamma(t)=\left\|z_{t}\right\|^{2}+\varepsilon\left[\left(z, z_{t}\right)+\frac{1}{2}\|\nabla z\|^{2}\right],
$$

for $\varepsilon>0$ small enough. Then, there exists a positive constants $C_{i}$ such that

$$
C_{1}\left(\left\|z_{t}\right\|^{2}+\|\nabla z\|^{2}\right) \leq \Gamma(t) \leq C_{2}\left(\left\|z_{t}\right\|^{2}+\|\nabla z\|^{2}\right) .
$$

From (A29) and (A33), we have the estimation

$$
\frac{d}{d t} \Gamma(t)+\left\|\nabla z_{t}\right\|^{2} \leq C_{\varepsilon, R} \Gamma(t)
$$

Using Gronwall's inequality, we get that

$$
\left\|z_{t}(t)\right\|^{2}+\|\nabla z(t)\|^{2}+\int_{\tau}^{t}\left\|\nabla z_{t}(s)\right\|^{2} d s \leq C_{R, T}\left(\left\|\mathrm{z}_{t}(\tau)\right\|^{2}+\|\nabla z(\tau)\|^{2}\right),
$$

for all $t \in[\tau, T]$, which implies the desired conclusion in (20). By this inequality, we can prove the existence of weak solutions for initial data $\left(u_{\tau}^{0}, u_{\tau}^{1}\right) \in \mathscr{H}$. Indeed, we can choose a sequence $\left\{\left(u_{\tau}^{0, n}, u_{\tau}^{1, n}\right)\right\} \in\left(H^{2}(\Omega) \cap H_{0}^{1}(\Omega)\right) \times$ $L^{2}(\Omega)$ such that $\left(u_{\tau}^{0, n}, u_{\tau}^{1, n}\right) \longrightarrow\left(u_{\tau}^{0}, u_{\tau}^{1}\right)$ in $\mathscr{H}$. Owing to 
(20), the corresponding solutions $\left(u^{n}(t), u_{t}^{n}(t)\right)$ converge to functions $\left(u, u_{t}\right)$ in $L^{\infty}(\tau, T ; \mathscr{H})$. From the boundedness for $\left\{u_{t}^{n}\right\}$ in $L^{2}\left(\tau, T ; H_{0}^{1}(\Omega)\right)$ we also have weak convergence of $\left\{u_{t}^{n}\right\}$ to $u_{t}$ in the space $L^{2}\left(\tau, T ; H_{0}^{1}(\Omega)\right)$. This implies that $u(t)$ is a weak solution of Problem (1). By (19), this solution is unique.

Step 3. For the proof of smoothness properties stated in (18), we use the same method as $[18,38]$. As usual, the argument below can be justified by considering Galerkin approximations. Set $v=u_{t}$ and differentiate (1) with respect to time. This yields

$$
v_{t t}-\Delta v_{t}-\phi\left(\|\nabla u\|^{2}\right) \Delta v-2 \phi^{\prime}\left(\|\nabla u\|^{2}\right) \Delta u\left(\nabla u, \nabla u_{t}\right)+f^{\prime}(u) v=h_{t} .
$$

Multiplying the above equation by $v$, we obtain that

$$
\begin{aligned}
& \frac{d}{d t}\left[\left(v, v_{t}\right)+\frac{1}{2}\|\nabla v\|^{2}\right]+\phi\left(\|\nabla u\|^{2}\right)\|\nabla v\|^{2}+\left(f^{\prime}(u) v, v\right) \\
& \quad \leq\left\|v_{t}\right\|^{2}+C_{R}|(\nabla u, \nabla v)|^{2}+\left(h_{t}, v\right) .
\end{aligned}
$$

This implies that

$$
\frac{d}{d t}\left[\left(v, v_{t}\right)+\frac{1}{2}\|\nabla v\|^{2}\right] \leq\left\|v_{t}\right\|^{2}+C_{1}\|\nabla v\|^{2}+\left\|h_{t}(t)\right\|^{2}
$$

Multiplying the above equation by $\mathscr{A}^{-1} v_{t}$ with $\mathscr{A}=-\Delta$ and using Young's inequality, we obtain that

$$
\frac{d}{d t}\left\|\mathscr{A}^{-1 / 2} v_{t}\right\|^{2}+\left\|v_{t}\right\|^{2} \leq C_{R}\left(\|\nabla v\|^{2}+\left\|h_{t}(t)\right\|^{2}\right) .
$$

Denote

$$
Y(t)=\left\|\mathscr{A}^{-1 / 2} v_{t}\right\|^{2}+\varepsilon\left[\left(v, v_{t}\right)+\frac{1}{2}\|\nabla v\|^{2}\right],
$$

then we have that

$a_{1}\left(\left\|\mathscr{A}^{-1 / 2} v_{t}\right\|^{2}+\|\nabla v\|^{2}\right) \leq Y(t) \leq a_{2}\left(\left\|\mathscr{A}^{-1 / 2} v_{t}\right\|^{2}+\|\nabla v\|^{2}\right)$,

for some positive constants $a_{i}$ depending on $\varepsilon$. Due to (A40) and (A41), it is apparent that

$$
\frac{d Y(t)}{d t}+\frac{1}{2}\left\|v_{t}\right\|^{2} \leq C_{3}\|\nabla v\|^{2}+C_{4}\left\|h_{t}(t)\right\|^{2} \leq \tilde{C}_{3} Y(t)+C_{4}\left\|h_{t}(t)\right\|^{2} .
$$

Multiplying (A44) by $(s-\tau)^{2}$, we get that

$$
\begin{aligned}
\frac{d}{d s}\left((s-\tau)^{2} Y(s)\right)+\frac{(s-\tau)^{2}}{2}\left\|v_{t}(s)\right\|^{2} \leq 2(s-\tau) Y(s) \\
+\tilde{C}_{3}(s-\tau)^{2} Y(s)+C_{4}(s-\tau)^{2}\left\|h_{t}(s)\right\|^{2}
\end{aligned}
$$

It is easy to know

$$
\begin{gathered}
2(s-\tau) Y(s) \leq 1+(s-\tau)^{2} Y^{2}(s) \leq 1+(s-\tau)^{2} \\
\cdot a_{2}\left(\left\|\mathscr{A}^{-1 / 2} v_{t}\right\|^{2}+\|\nabla v\|^{2}\right) Y(s) .
\end{gathered}
$$

Since

$$
\mathscr{A}^{-1} u_{t t}=-u_{t}-\phi\left(\|\nabla u\|^{2}\right) u-\mathscr{A}^{-1}(f(u)-h),
$$

one can see that $\left\|\mathscr{A}^{-1} v_{t}\right\| \leq C_{R}(1+\|h(\cdot, s)\|)$. Using $\left\|\mathscr{A}^{-1 / 2} v_{t}\right\|^{2} \leq C\left\|\mathscr{A}^{-1} v_{t}\right\| \cdot\left\|v_{t}\right\|$ and Young's inequality, we get

$$
\begin{aligned}
& \frac{d}{d s}\left((s-\tau)^{2} Y(s)\right)+\frac{(s-\tau)^{2}}{4}\left\|v_{t}(s)\right\|^{2} \leq C_{5}(s-\tau)^{2} \\
& \cdot\left(1+\left\|\nabla u_{t}\right\|^{2}\right) Y(s)+C_{6}(s-\tau)^{2}\left(\|h(\cdot, s)\|^{2}+\left\|h_{t}(s)\right\|^{2}\right) .
\end{aligned}
$$

By Gronwall's inequality and (16), one can find

$$
(t-\tau)^{2} Y(t) \leq C_{R, T}\left(1+\|h(\cdot, s)\|^{2}+\left\|h_{t}(s)\right\|^{2}\right) .
$$

This implies (18). The proof is completed.

\section{Data Availability}

All data used to support the findings of this study are included within the article.

\section{Conflicts of Interest}

The authors declare that they have no conflicts of interest.

\section{Acknowledgments}

This work was partly supported by the NSFC Grant 11801071 and the Scientific Research Project of Nanjing Xiaozhuang University (2017NXY53).

\section{References}

[1] G. Kirchhoff, Vorlesungen über Mechanik, Teubner, Sluttgart, 1883.

[2] A. Arosio and S. Spagnolo, "Global solutions to the Cauchy problem for a nonlinear hyperbolic equation, nonlinear partial differential equations and their applications," in Coll "ege de Frances seminar, vol. VI (Paris, 1982/1983), pp. 1-26, Res. Notes in Math.,109 Pitman, Boston, MA, 1984.

[3] S. Berstein, "Sur une classe d'e' quations fonctionelles aux de' rive' es," Izvestiya Akademii Nauk SSSR. Seriya Matematicheskaya, vol. 4, pp. 17-26, 1940. 
[4] P. D’Ancona and S. Spagnolo, "On an abstract weakly hyperbolic equation modelling the nonlinear vibrating string," in Developments in Partial Differential Equations and Applications to Mathematical Physics (Ferrara, 1991), pp. 27-32, Plenum, New York, 1992.

[5] P. D'Ancona and S. Spagnolo, "Global solvability for the degenerate Kirchhoff equation with real analytic data," Inventiones Mathematicae, vol. 108, pp. 247-262, 1992.

[6] P. D'Ancona and S. Spagnolo, "A class of nonlinear hyperbolic problems with global solutions," Archive for Rational Mechanics and Analysis, vol. 124, no. 3, pp. 201-219, 1993.

[7] J. M. Greenberg and S. C. Hu, "The initial value problem for a stretched string," Quarterly of Applied Mathematics, vol. 38, no. 3, pp. 289-311, 1980.

[8] T. Matsuyama and M. Ruzhansky, "Global well-posedness of Kirchhoff systems," Journal de Mathématiques Pures et Appliquées, vol. 100, no. 2, pp. 220-240, 2013.

[9] T. Yamazaki, "Global solvability for the Kirchhoff equations in exterior domains of dimension three," Journal of Differential Equations, vol. 210, no. 2, pp. 290-316, 2005.

[10] E. H. De Brito and J. Hale, "The damped elastic stretched string equation generalized: existence, uniqueness, regularity and stability," Applicable Analysis, vol. 13, no. 3, pp. 219233, 1982.

[11] M. Ghisi and M. Gobbino, "Global existence and asymptotic behavior for a mildly degenerate dissipative hyperbolic equation of Kirchhoff type," Asymptotic Analysis, vol. 40, no. 1, pp. 25-36, 2004.

[12] M. Ghisi, "Global solutions for dissipative Kirchhoff strings with non-Lipschitz nonlinear term," Journal of Differential Equations, vol. 230, no. 1, pp. 128-139, 2006.

[13] K. Nishihara and Y. Yamada, "On global solutions of some degenerate quasilinear hyperbolic equations with dissipative terms," Fako de l'Funkcialaj Ekvacioj Japana Matematika Societo, vol. 33, pp. 151-159, 1990.

[14] K. Ono, "On global existence, asymptotic stability and blowing up of solutions for some degenerate nonlinear wave equations of Kirchhoff type with a strong dissipation," Mathematicsl Methods in the Applied Sciences, vol. 20, pp. 151-177, 1997.

[15] Y. Yamada, "On some quasilinear wave equations with dissipative terms," Nagoya Mathematical Journal, vol. 87, pp. 1739, 1982.

[16] K. Nishihara, "Degenerate quasilinear hyperbolic equation with strong damping," Fako de l'Funkcialaj Ekvacioj Japana Matematika Societo, vol. 27, pp. 125-145, 1984.

[17] M. M. Cavalcanti, V. N. D. Cavalcanti, J. S. P. Filho, and J. A. Soriano, "Existence and exponential decay for a KirchhoffCarrier model with viscosity," Journal of Mathematical Analysis and Applications, vol. 226, no. 1, pp. 40-60, 1998.

[18] V. Kalantarov and S. Zelik, "Finite-dimensional attractors for the quasi-linear strongly-damped wave equation," Journal of Differential Equations, vol. 247, no. 4, pp. 1120-1155, 2009.

[19] M. Nakao, "An attractor for a nonlinear dissipative wave equation of Kirchhoff type," Journal of Mathematical Analysis and Applications, vol. 353, pp. 652-659, 2009.

[20] M. Nakao and Z. Yang, "Global attractors for some quasilinear wave equations with a strong dissipation," Advances in Mathematical Sciences and Applications, vol. 17, pp. 89-105, 2007.

[21] Z. J. Yang, P. Y. Ding, and Z. M. Liu, "Global attractor for the Kirchhoff type equations with strong nonlinear damping and supercritical nonlinearity," Applied Mathematics Letters, vol. 33, pp. 12-17, 2014.

[22] Z. J. Yang and Y. Wang, "Global attractor for the Kirchhoff equation with a strong dissipation," Journal of Differential Equations, vol. 249, pp. 3258-3278, 2010.

[23] Z. J. Yang and X. Li, "Finite-dimensional attractors for the Kirchhoff equation with a strong dissipation," Journal of Mathematical Analysis and Applications, vol. 375, no. 2, pp. 579-593, 2011.

[24] H. L. Ma, J. Zhang, and C. K. Zhong, "Global existence and asymptotic behavior of global smooth solutions to the Kirchhoff equations with strong nonlinear damping," Discrete and Continuous Dynamical Systems - Series B, vol. 24, no. 9, pp. 4721-4737, 2019.

[25] I. Chueshov, "Long-time dynamics of Kirchhoff wave models with strong nonlinear damping," Journal of Differential Equations, vol. 252, no. 2, pp. 1229-1262, 2012.

[26] H. L. Ma, J. Zhang, and C. K. Zhong, "Attractors for the degenerate Kirchhoff wave model with strong damping: existence and the fractal dimension," Journal of Mathematical Analysis and Applications, vol. 484, no. 1, article 123670, 2020.

[27] T. Caraballo, A. N. Carvalho, J. A. Langa, and F. Rivero, "Existence of pullback attractors for pullback asymptotically compact processes," Nonlinear Analysis, vol. 72, no. 3-4, pp. 1967-1976, 2010.

[28] T. Caraballo, P. E. Kloeden, and J. Real, "Pullback and forward attractors for a damped wave equation with delays," Stochastics and Dynamics, vol. 4, no. 3, pp. 405-423, 2004.

[29] T. Caraballo, G. Łukaszewicz, and J. Real, "Pullback attractors for asymptotically compact non-autonomous dynamical systems," Nonlinear Analysis: Theory, Methods \& Applications, vol. 64, no. 3, pp. 484-498, 2006.

[30] A. N. Carvalho, J. A. Langa, J. C. Robinson, and A. Suárez, "Characterization of non-autonomous attractors of a perturbed infinite-dimensional gradient system," Journal of Differential Equations, vol. 236, no. 2, pp. 570-603, 2007.

[31] P. E. Kloeden, "Pullback attractors of non-autonomous semidynamical systems," Stochastics and Dynamics, vol. 3, no. 1, pp. 101-112, 2003.

[32] C. Y. Sun, D. M. Cao, and J. Q. Duan, "Non-autonomous dynamics of wave equations with nonlinear damping and critical nonlinearity," Nonlinearity, vol. 19, pp. 2645-2665, 2006.

[33] Y. H. Wang and C. K. Zhong, "Upper semicontinuity of pullback attractors for nonautonomous Kirchhoff wave models," Discrete and Continuous Dynamical Systems, vol. 7, pp. 3189-3209, 2013.

[34] Y. N. Li and Z. J. Yang, "Robustness of attractors for nonautonomous Kirchhoff wave models with strong nonlinear damping," Applied Mathematics \& Optimization, vol. 84, no. 1, pp. 245-272, 2021.

[35] Q. F. Ma, S. H. Wang, and C. K. Zhong, "Necessary and sufficient conditions for the existence of global attractors for semigroups and applications," Indiana University Mathematics Journal, vol. 51, pp. 1541-1559, 2002.

[36] Y. J. Wang, C. K. Zhong, and S. F. Zhou, "Pullback attractors of nonautonomous dynamical systems," Discrete and Continuous Dynamical Systems, vol. 16, pp. 587-614, 2006.

[37] J. Lions, Quelques Méthodes de Résolution des Problèmes Aux Limites Non Linéaires, Dunod, Paris, 1969.

[38] A. V. Babin and M. I. Vishik, Attractors of Evolution Equations, North-Holland, Amsterdam, 1992. 